\title{
Bose-Einstein Condensates in Optical Lattices
}

\author{
W. Wasilewski ${ }^{a}$, M. TRIPPEnBaCH ${ }^{a}$ AND K. RzążEWSKI ${ }^{b}$ \\ ${ }^{a}$ Institute of Experimental Physics, Optics Division \\ Warsaw University, Hoża 69, 00-681 Warsaw, Poland \\ ${ }^{b}$ Center for Theoretical Physics, Polish Academy of Sciences \\ al. Lotników 32/46, 02-668 Warsaw, Poland
}

\begin{abstract}
We study an ultracold dilute gas of bosonic atoms in an optical lattice induced by intersecting laser beams. As a first approximation we neglect confining potential and atom-atom interactions. In this case the GrossPitaevskij equation reduces to simple Mathieu equation. Upon choosing periodic boundary conditions this problem has well known periodic solution. This simple picture allows to demonstrate localization of the wave packet and formation of the band structure. We calculate spectrum of the excited states as a function of the strength of modulating potential and using a standard adiabaticity criterion we predict the most efficient way to ramp up optical lattice, without higher state excitation. Finally, we discuss the influence of the atom-atom interaction (nonlinearity) on the adiabaticity of the process.
\end{abstract}

PACS numbers: 03.75.Fi, 03.67.Hk, 03.67.Lx, 71.35.Lk

\section{Introduction}

Recently developed systems of trapped ultracold atomic gases open a new field for study of many-particle physics [1]. This includes the investigation of the Bose-Einstein condensation of interacting Bose particles, leading to macroscopic quantum states or matter waves. One of the most interesting aspects there is the interaction of Bose-Einstein condensates (BEC) with optical waves, and among them trapping of atoms in the so-called optical lattices [3]. The spatially periodic intensity variation in the standing wave of laser light translates, by means of $\mathrm{AC}$ Stark effect, into spatially periodic potential felt by atoms moving in this standing wave. The depth of such an optical potential is proportional to the square of the Rabi frequency of the atomic transition involved, and inversely proportional to the 
detuning of the laser from that transition [4]. Interaction of standing waves with BEC opened a wide range of applications, from Bragg scattering (out-coupling) [5] of atomic wave packets, Bragg spectroscopy [6], nonlinear atomic optical phenomena [7], coherent control of the atomic wave packets [8], to the recent studies of tunneling dynamics in optical lattices [9]. Recently, many theoretical and experimental groups investigate an idea of applying optical lattices for implementation of logical quantum gates. It is crucial for these applications that the optical lattices, which are in most cases dynamically imposed over an existing condensate, keep the condensate in the ground state of the system, or in other words the process of ramping up of the lattice should remain adiabatic.

Adiabaticity in nonlinear systems can be studied in various regimes. Denoting $\tau_{\mathrm{AD}}$ as the quantum-mechanical linear adiabatic timescale determined in terms of the difference of the (linear) energy eigenvalues [2] and $\tau_{\mathrm{NL}}$ the nonlinear timescale [10], the simplest regime is one in which the duration of the dynamical process being studied, $T$, satisfies the condition, $\tau_{\mathrm{AD}} \ll T \ll \tau_{\mathrm{NL}}$. In this case, adiabaticity is insured by the adiabatic theorem due to the first inequality, and nonlinearity plays only a secondary role in the dynamics due to the second inequality. Hence, the dynamics is in principle adiabatic. In this paper we first study the dynamics in relatively short timescale, find the most efficient dependence of the optical lattice ramp to preserve adiabaticity and discuss coupling between different states in the regime when nonlinearity is a small effect. We also point out the effects that nonlinearity might have on the dynamics of optical lattices on the longer timescales, and at the end we give a brief discussion and summary on the general question of adiabaticity in the nonlinear systems.

This study was inspired by two experiments: at NIST [11] and Yale [12]. The central question of this paper is how fast and along what ramp one should increase the intensity of the standing wave in order to preserve adiabaticity of the process. Adiabaticity in this case means that at each instant of time, our wave function remains an eigenstate of the instantaneous Hamiltonian. The answer is found in the form of the prescription for the functional dependence of the light intensity to minimize the effect of decoherence and excitation of the condensate during the growth of the optical lattice (rising of the light potential). This prescription is

derived directly from the adiabatic condition. We present numerical results for the one-dimensional condensate dynamics in the growing light potential and discuss what is the effect of mean-field interaction.

\section{Simple model for the BEC in the standing wave. Single well case}

Bose-Einstein condensate in standing wave is described by the GrossPitaevskij (GP) equation:

$$
i \hbar \frac{\partial \psi}{\partial t}=-\frac{\hbar^{2}}{2 m} \frac{\partial^{2} \psi}{\partial x^{2}}+m \omega^{2} x^{2} \psi+\frac{4 \pi \hbar^{2} a N}{m}|\psi|^{2} \psi+\frac{\hbar \Omega^{2}}{2 \Delta} \sin (k x) \psi,
$$


which we rewrite in the dimensionless form $\left(x=x_{0} \zeta, t=t_{0} \tau\right)$

$$
\begin{aligned}
\mathrm{i} \frac{\partial \psi}{\partial \tau} & =-\frac{\partial^{2} \psi}{\partial \xi^{2}}+\underbrace{8 \pi^{4}\left(\frac{\hbar \omega}{E_{\mathrm{R}}}\right)^{2}}_{\eta} \xi^{2} \psi+\underbrace{\frac{20 N}{\pi} \frac{x_{0} a_{0}}{\omega_{0}^{2}}}_{U}|\psi|^{2} \psi \\
& +\underbrace{4 \pi^{2} \frac{\hbar \Omega^{2} / \Delta}{E_{\mathrm{R}}}}_{A} \sin \left(k x_{0} \xi\right) \psi,
\end{aligned}
$$

where $m$ is atomic mass, $\omega$ is the trap frequency, $\omega_{0}$ is a Thomas-Fermi radius of the condensate, $x_{0}=\lambda / 2, a_{0}$ is the scattering length, $N-$ number of atoms in the condensate, $\Omega$ is the Rabi frequency of the optical transition of the optical lattice, and $\Delta$ is the detuning from the resonance of this transition. $E_{\mathrm{R}}$ is the so-called recoil energy and is equal to $E_{\mathbf{R}}=\hbar^{2} k^{2} / 2 m$. In reduced (dimensionless) units $\eta$ is strength of harmonic potential, $U$ - strength of nonlinearity and $A$ is a depth of the modulation of the optical lattice.

The ground state of the wave function can be obtained from the above GP equation via an imaginary time method, which is an iterative procedure consisting of steps of propagation of the wave function according to Eq. (1) with imaginary time and renormalizing it. Here, for most part, we choose different approach to study energy structure of the system. We neglect the trapping potential and nonlinearity in the Gross-Pitaevskij equation and reduce the problem to much simpler and elementary form, studied in many textbooks. This crude approximation is justified by the fact that in most situations the strength of the optical potential is many orders of magnitude bigger than that of nonlinear or harmonic trapping potential. We will present more detailed justification of this approximation below.

In absence of trapping potential and nonlinearity the only natural length scale is the wavelength of light forming the lattice: $\lambda=k / 2 \pi$. If we choose the half of the wavelength as a unit of length $\left(x_{0}\right)$ and impose additional condition that the coefficient in the kinetic energy operator, in front of the spatial derivative, to be equal to unity we introduce unit of time $\left(t_{0}=2 m x_{0}^{2} / \hbar\right)$. To further reduce our problem to the single cell of the optical lattice we impose periodic boundary conditions that wave function $\psi(0)=\psi(1)$. Upon imposing above mentioned units and conditions, Eq. (1) is reduced to the Mathieu equation [13]:

$$
E \psi(\xi)=\frac{\partial^{2} \psi(\xi)}{\partial \xi^{2}}+A \cos (2 \pi \xi) \psi(\xi), \quad \xi \in[0,1]
$$

We first study the localization of the wave packet in the optical lattice using our simple one-dimensional model. We assume that one-dimensional optical lattice is formed by the standing wave, but our model can be easily extended to include standing-moving potentials and presence of the trapping forces. In the experiment, wavelength of the laser light is much smaller than the size of the BEC condensate. For example in the NIST experiment Thomas-Fermi radius of the condensate corresponds to approximately 100 wavelengths, which was equal to $589 \mathrm{~nm}$. This, 
as we mentioned above, justifies our dropping of the confining potential. We solve Eq. (3) with periodic boundary conditions. Since $\psi(0)=\psi(1)$, hence we only picture the wave function within one wavelength region. We seek the solutions in the form of a power series of periodic functions: $\psi(\xi)=\sum_{l=-\infty}^{+\infty} C_{l} \exp (2 \pi l \xi)$. Upon substituting this expansion into Eq. (3) we obtain a set of recurrent equations for coefficients $C_{l}$. One can rewrite this recurrence for the ratios of $C_{l} / C_{l-1}$ and $C_{l} / C_{l+1}$, solve two sets of equations starting from, say $C_{-L}$ and $C_{L}$, and obtain the quantization condition for the energy by demanding that two solutions meet at $C_{0}$ (one gets solutions in the form of continuous fractions - see [13]). We have chosen slightly different formulation. We introduce $L$-th order approximation in which $C_{L}$ and $C_{-L}$ coefficients are equal to zero, write down a set of linear coupled equation for $C_{l} ;-L<l<L$ coefficients and solve the eigenvalue problem

$$
\begin{gathered}
\left(\begin{array}{ccccccc}
\ddots & \vdots & \vdots & \vdots & \vdots & \vdots & \ddots \\
\ldots & 4 \pi^{2}(2)^{2}-E & A & 0 & 0 & 0 & \ldots \\
\cdots & A & 4 \pi^{2}(1)^{2}-E & A & 0 & 0 & \ldots \\
\cdots & 0 & A & -E & A & 0 & \ldots \\
\cdots & 0 & 0 & A & 4 \pi^{2}(-1)^{2}-E & A & \ldots \\
\cdots & 0 & 0 & 0 & A & 4 \pi^{2}(-2)^{2}-E & \ldots \\
\ddots & \vdots & \vdots & \vdots & \vdots & \ddots
\end{array}\right) \\
\quad\left(\begin{array}{c}
\vdots \\
C_{2} \\
C_{1} \\
C_{0} \\
C_{-1} \\
C_{-2} \\
\vdots
\end{array}\right)=0 \\
\end{gathered}
$$

By solving the eigenvalue problem, (4), we obtain energies of the ground and first $2 L$ excited states. This method is very efficient, and convergence (value of $L$ required) depends on the value of the potential $A$. In Fig. 1 we present results of our calculations for three different values of the potential $A$. For $A=1$, light potential is too weak to force any localization of the wave function, which in this case remains almost constant. The value of the constant wave function is found from the normalization condition. With increasing value of the light potential the wave function becomes more and more localized around the antinode of the potential. Light is pushing out atoms from the high to the low amplitude regions. At the value of $A=50$ we clearly see the effect of localization, and at $A=500$ wave function drops to zero at the boundaries. The latter case will correspond to modulation strength of order of $15 \mathrm{E}_{\mathrm{R}}$ for sodium atoms in the NIST experiment, when the separation is strong between peaks of the wave function. 


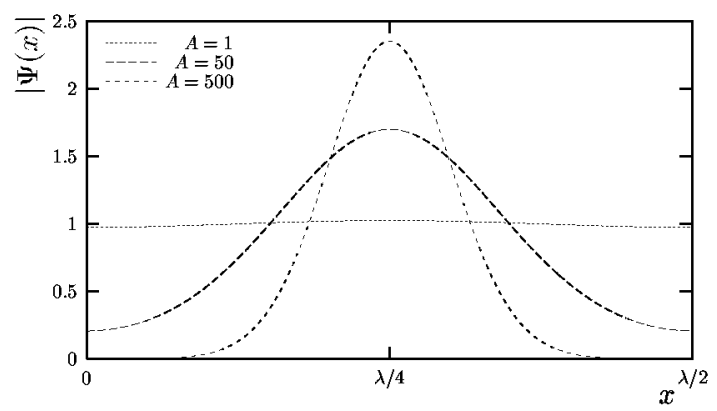

Fig. 1. Ground states of the condensate wave function for various intensities of periodic optical potential, without nonlinearity.

The advantage of studying this model is twofold: first, we have simple Mathieu equation replacing GP equation and we can solve for eigenstates (they are given in the form of continuous fractions, or eigenvectors of Eq. (4)) and, second, we can easily get information about excited states, their wave functions, and energies. It is important, as we will show later, for the description of dynamic processes, for example when amplitude $A$ is a function of time and is essential if one is interested in such aspects as adiabaticity.

As Eq. (3) is as a matter of fact the special case of the Gross-Pitaevskij equation and one can find the value of eigenenergy and corresponding eigenstate also by different means. Here we also used imaginary time method. We checked that the results obtained both ways agreed very well. Imaginary time method has another advantage, namely it can be easily extended to the study of the effects of atom-atom interaction, simply by adding a nonlinear term to the Gross-Pitaevskij equation. One expects that the atom-atom interaction becomes important when the size of the nonlinear term is of the same magnitude as the size of the light potential term. The former may be estimated, at least for big number of atoms in the condensate, by the value of the chemical potential. The results of our studies are summarized in Fig. 2. We present the ground state wa ve functions for four different values of $U_{0}$ for $A=50$. At $U_{0}=50$ distortion of the wave function becomes noticeable and the localization is nearly completely washed out at $U_{0}=250$. Surely for higher values of $A$ one needs higher value of nonlinearity to compete with the light potential. The main conclusion from the above discussion is that at typical atom densities used in the atomic traps, when the intensity of the laser light forming the optical lattice is just starting to form a noticeable structure on the condensate, the atom-atom interaction may be neglected. For a sodium condensate consisting of $10^{6}$ atoms and trap frequency of $50 \mathrm{~Hz}$ already at the intensity of the order of $50 \mathrm{~mW} / \mathrm{cm}^{2}$ it is virtually impossible to distinguish between linear and nonlinear wave functions.

Imaginary time method is very efficient in obtaining a ground state and its energy, but is not very efficient for finding excited states. It is much easier 


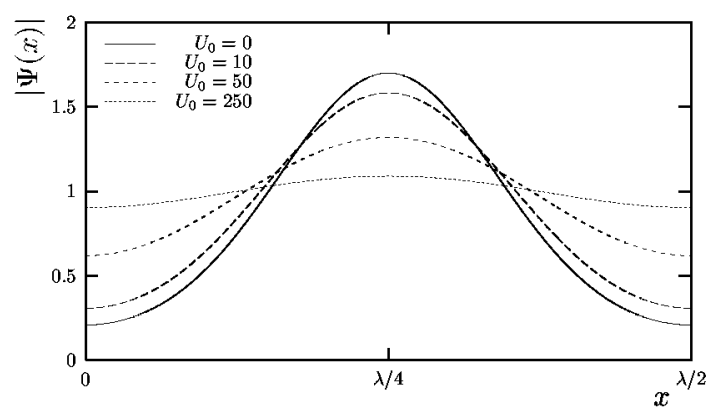

Fig. 2. Ground states of the condensate wave function for constant intensity $(A=50)$, with various nonlinearities (atom-atom interaction strength).

to find excited states energies and corresponding wave functions by the method used in the theory of deterministic chaos, namely by evolving the wave function from some arbitrarily chosen initial state in the configuration space, finding the value of the wave function at each point as a function of time $\psi\left(x_{0}, t\right)$ and taking Fourier transform in $t$. As we expect, we find the same maxima for all values of $x_{0}$ provided we started from initial state that had "admixtures" of all excited states (we use the word "admixtures" in the quotation mark, since in the nonlinear case the superposition principle does not hold) and they correspond to energies of the ground and excited states. One can even reconstruct the shape of wave functions corresponding to these energies.

We conclude this section with the short discussion of the multicell case. So far we considered the single cell case, namely there was only one well of the optical lattice over the size of the wave function. Upon expanding of the wave function, we need to consider more wells of the lattice. This is the case in all of the experiments performed so far. A number of wells varies between 10 and 100 wells. The energy-level structure that we consider here for the single well case, would have to be generalized to include multiwell case, namely each of the state would have to be replaced by a band of states and number of states in the band being equal to the number of wells over the size of the condensate. These bands are called Brillouin zones, and we will use this term later in the discussion of the nonadiabaticity.

\section{Time varying light potentials and adiabaticity}

In the previous section we investigated stationary problem by finding the spectrum of energies and wave functions for certain value of the laser light intensity and therefore a fixed optical lattice. We now consider the case of the time-varying amplitude of the light forming optical lattice. It is no longer a stationary problem and we need to replace Eq. (3) with the evolution equation. It is more complicated problem, since in general it is associated with generating excitation in the condensate. Here however we will concentrate on the switching-on effects and the 
adiabaticity issues. Namely, we will pose the question how fast and with what functional dependence of $A(t)$ one can increase the intensity of the standing wave and still preserve adiabaticity of the process. As we already mentioned in the introduction, this problem is very important in the context of forming optical lattices. To built quantum gates from atoms in the lattice one needs very high fidelity of the system, and even small amount of excitation can effect operational capacity of such a gate. If we want to control atoms in the lattice, we certainly want to form the lattice carefully enough to preserve initial coherence of the condensate. Here a very objective test would be to compare how we change properties of the wave function by rising light potential to certain value and then slowly turning it back to zero value. Results of such a test will strongly depend on how functional dependence of $A(t)$ is. As a mean of our study we use initially the same simple model of the Hamiltonian with periodic boundary conditions, and then we will discuss effects of nonlinearity and eventually trapping harmonic potential by switching to the Gross-Pitaevskij equation. We call the process of varying the strength of the modulation of the optical lattice "adiabatic" if at each instant in time, our wave function remains an eigenstate of the instantaneous Hamiltonian. Hence adiabaticity should assure that if the Hamiltonian varies in time slowly enough, the transition to the instantaneous excited states should remain small. In more rigorous terms adiabatic condition may be expressed by the relation

$$
\frac{1}{\omega_{01}}\left\langle 0\left|\frac{\mathrm{d}}{\mathrm{d} t} H(t)\right| 1\right\rangle \ll \hbar \omega_{01}
$$

where $\hbar \omega_{01}$ stands for the energy difference between the ground and excited states. In our case varying part of the Hamiltonian is only the light potential and $\mathrm{d} H / \mathrm{d} t=$ $\mathrm{d} A / \mathrm{d} t$. What appears on the right hand side is the difference between the ground and excited state energy. In our simple model this energy difference is a nonlinear function of $A$. We will call this functional dependence $\Delta E(A)$ and for our simple model it is shown in Fig. 3 in the $\log -\log$ scale in the range of $A$ from 0 to 1000. The upper limit corresponds to very strong localization of the wave function and the depth of the lattice of $20 E_{\mathrm{R}}$ for the case of sodium. The energy gap grows initially very slowly to rise more abruptly at the range of $A$ of order 100 . The information of $\Delta E(A)$ may be used for finding the most efficient way of forming optical lattice. It tells us for what $A(t)$ the growth of $A$ can be made in the shortest time still fulling adiabaticity condition. Upon substituting $\omega_{01}$ in Eq. (5) we obtain (in reduced units)

$$
\left\langle 0\left|\frac{\mathrm{d}}{\mathrm{d} t} A\right| 1\right\rangle \ll \Delta E(A)^{2}
$$

If this criterion is satisfied during each instant of time during the evolution we guarantee that the transfer of the population to the excited states is negligible. And we can use this inequality to find a functional dependence of $A(t)$ that guarantees the adiabaticity of the process. Namely, we will put a small factor $\gamma \ll 1$ on 


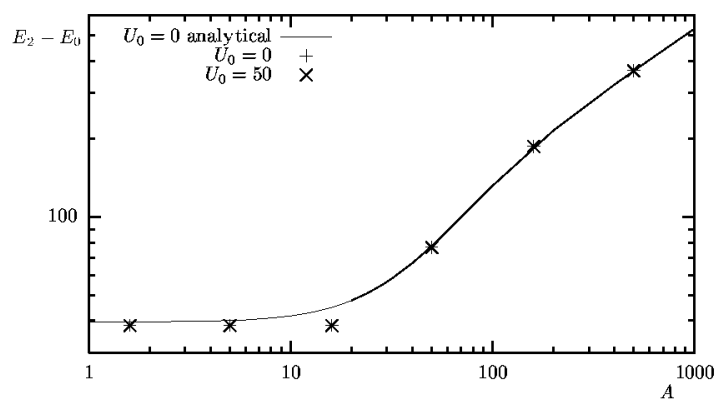

Fig. 3. Energy gap between ground and first possibly excited by the periodic optical potential level vs. varying intensity of the optical potential $A$. The continuous curve was obtained by solving Eq. (3), while points correspond to the results obtained from propagation with constant Hamiltonian without and with nonlinearity (points overlap).

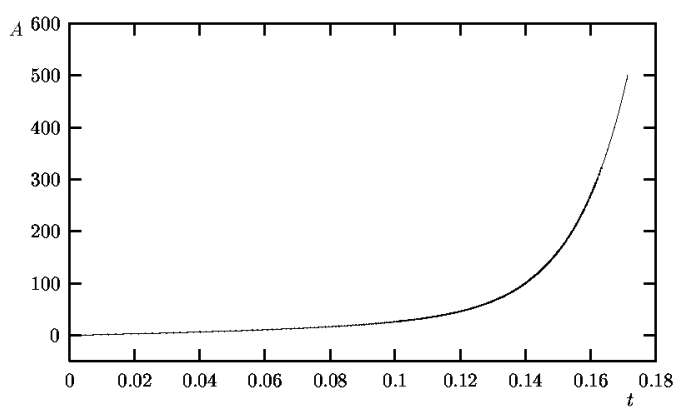

Fig. 4. Results of numerical integration of equation $\mathrm{d} A / \mathrm{d} t=\Delta E^{2}$ with initial $A=0$ and final $A=500$.

the right hand side of the inequality and replace $\ll$ with an equal sign, this way obtaining an ordinary differential equation for $A(t)$ :

$$
\left\langle 0\left|\frac{\mathrm{d}}{\mathrm{d} t} A\right| 1\right\rangle=\gamma \Delta E(A)^{2}
$$

A solution of this equation is presented in Fig. 4. Both time and $A$-strength of the lattice modulation are presented in the reduced units. Initial variation of $A(t)$ is determined by the level spacing of $4 E_{\mathrm{R}}$, which corresponds to the case of no lattice present. Then the pace can be increased, because level spacing between ground and excited state is a monotonically growing function of $A$. We only included excitation of the first state coupled by the optical potential, but the transfer of population to the higher excited states is negligible if we satisfy Eq. (7). We also checked by numerical simulation that even with the value of the $\gamma=0.1$ after application of the ramp shown in Fig. 4 the wave function remains almost perfectly adiabatic, with the transfer to the excited state less than $0.1 \%$. 


\section{Testing adiabaticity. \\ Two types of excitation during optical lattice turn-on}

We will now discuss what forms of nonadiabaticity one can expect if the lattice potential is ramped up too fast or on the ramp that is much different than the one found in the previous section. One can check in the numerical simulations (and this is also seen in the experiment) that if the ramp up of the light potential is not too fast in the Fourier domain, the wave function consists of atoms with momenta centered around 0 and $\pm 2 \hbar k$. Since the value of $\hbar k$ for typical laser beams used for lattices is much bigger that the mean spread of the values of the momentum within the condensate, upon taking a Fourier transform of the wave function one sees a three-peak structure, corresponding to atoms moving with a verage 0 momentum, and average $\pm 2 \hbar k$. In the experiment this feature can be seen if after turning on the light potential, we suddenly drop it to zero (switch of the lasers forming a lattice suddenly) and wait for some time until the condensate will be separated into three parts corresponding to three different mean values of the momentum and finally we measure the population in each of the three wave packets. In our simulations in this part of the presentation, we used the full GP equation. Figure 5 shows the result of this simulation. The upper curve corresponds to the population of atoms in the 0 mean momentum peak, and lower curve corresponds to the population within $2 \hbar k$ peak. For each time two points on the curves would correspond to the certain value of $A(t)$. We have chosen the case when $A$ linearly increases from zero to the value of $14 E_{\mathbf{R}}$ (sodium condensate with

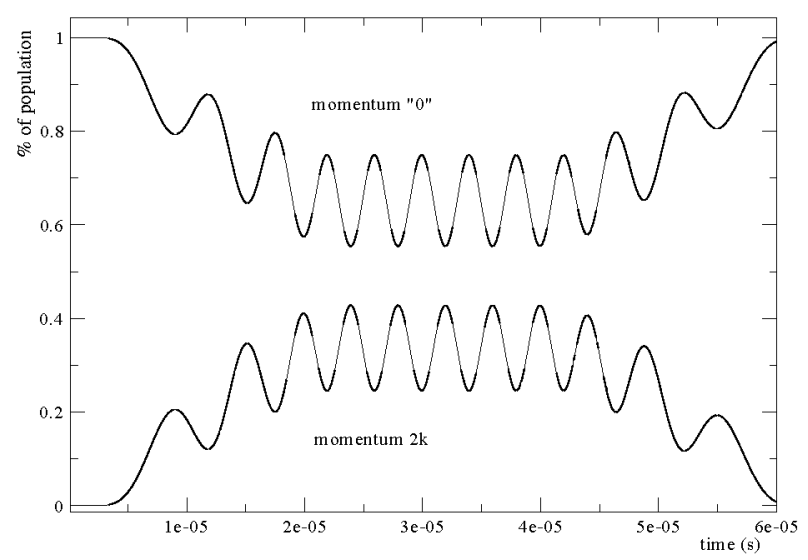

Fig. 5. Population of ground (upper curve) and first excited (lower curve) energy bands as a function of time. Between 0 and $20 \mu$ s the optical lattice was turned on on the linear ramp, then was up for $20 \mu$ s and finally turned off on the linear ramp during $20 \mu \mathrm{s}$. Excitation of the first energy band can be seen. Period of the oscillation corresponds to the energy difference between bands. 
$10^{5}$ atoms and trap frequency of $50 \mathrm{~Hz}$ ) over the time of $20 \mu \mathrm{s}$, stays constant for the next $20 \mu$ s and finally decreases linearly to zero again over the period of $20 \mu \mathrm{s}$. One distinctive feature in Fig. 5 is the oscillation that appears almost immediately after turn on of the lattice. The period of these oscillations is equal to the distance between ground and excited state discussed in the previous section. The period should be measured within the interval 20-40 $\mu$ s, when the strength of the lattice modulations corresponds to constant value of $14 E_{\mathrm{R}}$. The amplitude of these oscillations can be viewed as a measure of the nonadiabaticity. In the case of smooth and adiabatic process of dynamical light potential changing they will not be present and we tested it with the curve predicted in the previous section.

This type of excitation appears when the turn on process is nonadiabatic, both in the linear and nonlinear systems. It corresponds to the excitation of the state in the first Brillouin zone coupled by the light potential. In the linear case there is no coupling within each Brillouin zone. However, with nonlinear coupling present this situation changes. Namely, nonlinearity couples states within each Brillouin zone. We illustrate this process with the results presented in Fig. 6. It shows the phase of the wave function after the lattice has been slowly ramped up and down, on the same timescale as in Fig. 5, but along much smoother curve, upon which we have seen almost no oscillations due to the higher zone state excitation. Since the timescale of the dynamics was short in comparison with the condensate wave function expansion, the amplitude of the wave function was essentially the same as at the beginning, before imposing the lattice. At the same time during the evolution a spatial phase structure has been developed as we clearly see in Fig. 6 . This spatially dependent structure in the phase of the condensate is due to the nonlinear interaction. It starts to develop when the tunneling time between wells is

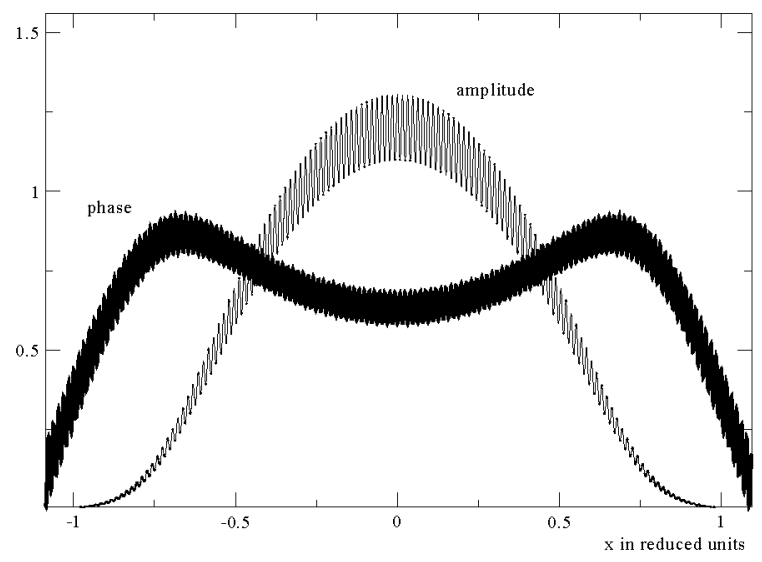

Fig. 6. Amplitude and phase of the condensate wave function after adiabatic optical lattice turn on and off. The nontrivial structure is due to the coupling between states in single band caused by nonlinearity. 
becoming comparable to the nonlinear time of the system, or in other words, when the spacing between levels within Brillouin zone becomes very small. We checked that leaving the trapping potential on during the increase in the lattice potential to some extent helps to remove the phase due to the compensation between harmonic potential and nonlinearity, but it does not go away completely. We will continue discussion of effects of the nonlinearity in the next section.

\section{The role of nonlinearity}

In our presentation we were mainly concerned with the case of $\tau_{\mathrm{AD}} \ll$ $T \ll \tau_{\mathrm{NL}}$, where $T$ is a duration of the dynamic process, $\tau_{\mathrm{AD}}$ is characteristic adiabatic time and $\tau_{\mathrm{NL}}$ is a characteristic time of the nonlinearity, as was presented in the introduction. But there is another interesting regime, when the dynamical timescale is the largest one in the problem, i.e., $\tau_{\mathrm{AD}}, \tau_{\mathrm{NL}} \ll \mathrm{T}$. This case applies, e.g., to the BEC experiments reported in Refs. $[12,14]$. In this case, the nonlinearity plays an essential role in the dynamics, and a relevant question is whether the dynamics can be adiabatic. In [15] a simple model of an atomic Bose-Einstein condensate in a box whose size varies with time was studied to determine the nature of adiabaticity in the nonlinear dynamics obtained within the Gross-Pitaevskii equation (the nonlinear Schrödinger equation). Analytical and numerical methods were used to determine the nature of adiabaticity in this nonlinear quantum system. It was shown that if the dynamics of the process is sufficiently slow it can be adiabatic.

Adiabaticity of soliton solutions of nonlinear wave equations have been extensively studied for slowly varying external conditions (for a review, see Ref. [16]). It has been shown that if the timescale $T$ of the variation of an external parameter is slow compared to the instantaneous nonlinear eigenvalue $\omega_{0}(t)$ of the nonlinear (time-independent) equation at time $t, \omega_{0}(t) T / 2 \pi \gg 1$, the dynamics can be adiabatic. This is a very similar criterion that was found in above mentioned study [15]. There are indications though that the case of optical lattices might be different.

A very interesting study of the nonlinearity of the BEC was shown in an experiment performed at Yale [12]. In the experiment Bose-Einstein condensed ${ }^{87} \mathrm{Rb}$ atoms in the $\left|F=2 ; M_{F}=2\right\rangle$ hyperfine state were confined in an array of optical traps in a gravitational field. The atoms were trapped at the antinodes of a vertically oriented red-detuned optical standing wave, which were separated by $\Delta z=\lambda / 2$, where $\lambda=840 \mathrm{~nm}$ is the wavelength of light used to confine the atoms. The depth of the optical potential was proportional to the intensity of the light; the intensity is initially zero and linearly increases with time. The initial BEC was cigar shaped with $10^{4}$ atoms in a static harmonic trap potential

$$
V_{\mathrm{ho}}(r)=\frac{m \omega_{z}}{2} z^{2}+\frac{m \omega_{x, y}}{2}\left(x^{2}+y^{2}\right)
$$


with frequencies $\omega_{z}=2 \pi \times 19 \mathrm{~Hz}$, and $\bar{\omega}=2 \pi \times 33 \mathrm{~Hz}\left(\bar{\omega}=\left(\omega_{x} \omega_{y} \omega_{z}\right)^{1 / 3}\right.$ with $\left.\omega_{x}=\omega_{y} \equiv \omega_{x y}\right)$. The light potential experienced by the atoms in the BEC was given by $V_{L}(z, t)=V_{0}(t)\left[1+\cos \left(2 k_{L} z\right)\right]$, where the well depth $V_{0}(t)=\left(250\left[E_{\mathrm{R}} / \mathrm{s}\right]\right) t$ was varied linearly with time [12]. The recoil energy is defined as $E_{\mathrm{R}}=\hbar^{2} k_{L}^{2} / 2 m$, $m$ is the atomic mass, and the photon wave vector is $k_{L}=2 \pi / \lambda$. The rate of increase in the light potential, $\left(250\left[E_{\mathrm{R}} / \mathrm{s}\right]\right)$, was sufficiently slow, that the dynamics of the BEC was found to be adiabatic for much of the turn-on. After some time, the harmonic potential and the light potential were switched off (dropped) releasing the atoms to fall under the influence of gravity. In the experiments reported in Ref. [12], the atoms are held in the optical lattice for a short time $(2.5 \mathrm{~ms})$ after switching off the harmonic potential, allowing the gravitational potential difference between wells to affect the phase difference between wells, and absorption images were taken $8 \mathrm{~ms}$ after the optical potential is turned off and the atoms begin to free fall. A surprising loss of contrast in the interference patterns was found when the optical lattice was rised slowly to the value at which there was essentially no tunneling between different wells. It was attributed to the random phase that arises between condensate wave packets located at different antinodes of the lattice.

The process of dynamic splitting of a condensate by an external time-varying potential was studied using a two-mode model within a variational ansatz to describe both internal and external evolution of the modes in Ref. [17]. It claims that the mean-field approximation based upon the GP equation will not give a good description of the splitting process due to a slow rise of a potential barrier which cuts off tunneling processes.

The results of this experiment were studied using one-dimensional GP equation, with parameters similar to those present in the experiment, and lead to very surprising conclusions [18]. It turned out that the adiabaticity was maintained throughout the course of the dynamics until the wave packets become well separated and the tunneling time becomes comparable to or larger than the nonlinear timescale. When the tunneling time became comparable with the nonlinear time quite suddenly the spatially varying phase of the wave function begins to grow significantly, and the spatially dependent variations increase as the well depth of the optical lattice increases. The study mentioned above shows that an additional condition is required; adiabaticity breaks down when the wave packets become well separated and the tunneling time becomes longer than the nonlinear time. The nature of this phenomenon and breakup of the mean-field approximation require further studies.

\section{Summary and conclusions}

We presented a study of the behavior of BEC atoms in an optical lattice induced by intersecting laser beams. As a first approximation we neglect confining potential and atom-atom interactions and show that in this case the 
Gross-Pitaevskij equation reduces to a simple Mathieu equation. Using this equation we study the adiabatic form of rising an optical lattice over the condensate. We found the most efficient adiabatic way to ramp up optical lattice, without transfer of the population from the ground state of the condensate to the excited states. Finally, we discussed the influence of the atom-atom interaction on the adiabaticity of the process and addressed the issue of the adiabaticity in the nonlinear systems in general.

M.T. would like to thank Yehuda B. Band and Paul S. Julienne for valuable discussions. This work was supported in part by grant of the State Committee for Scientific Research, no. 2/P03/B07819 (W.W. and M.T.), and Foundation for Polish Science (K.R.). Part of the calculations were carried out at the Interdisciplinary Center for Mathematical and Computational Modeling (ICM), Warsaw University.

\section{References}

[1] M.H. Anderson, J.R. Ensher, M.R. Matthews, C.E. Wieman, E. Cornell, Science 269, 198 (1995); C.C. Bradley, C.A. Sackett, R.G. Hulet, Phys. Rev. Lett. 75, 1687 (1995); K.B. Davis, M.O. Mewes, M.R. Andrews, N.J. van Druten, D.S. Durfee, D.M. Kurn, W. Ketterle, Phys. Rev. Lett. 75, 3969 (1995).

[2] A. Messiah, Quantum Mechanics, Vol. II, North-Holland, Amsterdam 1975, Ch. 17.

[3] D. Jaksch, C. Bruder, J.I. Cirac, C.W. Gardiner, P. Zoller, Phys. Rev. Lett. 81, 3108 (1998); D. van Oosten, P. van Stratten, H.T.C. Stoof, Phys. Rev. A 63, 053601 (2001).

[4] A.P. Kazantsev, G.I. Surdutovich, V.P. Yakovlev, Mechanical Action of Light on Atoms, World Sci., Singapore 1990.

[5] M. Kozuma, L. Deng, E.W. Hugley, J. Wen, R. Lutwak, K. Helmerson, S.L. Rolston, W.D. Phillips, Phys. Rev. Lett. 82, 871 (1999).

[6] J. Stenger, S. Inouye, A.P. Chikkatur, D.M. Stempler-Kuhn, D.E. Prichard, W. Ketterle, Phys. Rev. Lett. 82, 4569 (1998).

[7] L. Deng, E.W. Hugley, J. Wen, M. Trippenbach, Y. Band, P.S. Julienne, J.E. Smisarian, K. Helmerson, S.L. Rolston, W.D. Phillips, Nature 398, 218 (1999).

[8] P. Rudy, R. Ejnisman, N.P. Bigelow, Phys. Rev. Lett. 78, 4906 (1997); I.H. Deutsch, P.S. Jensen, Phys. Rev. A 57, 1978 (1998).

[9] S.K. Dutta, B.K. Teo, G. Reithel, Phys. Rev. Lett. 83, 1934 (1999).

[10] See for example G.P. Agrawal, Nonlinear Fiber Optics, Academic Press, New York 1989 or M. Trippenbach, Y.B. Band, Phys. Rev. A 56, 4242 (1997); Phys. Rev. A $\mathbf{5 7}, 4791$ (1998).

[11] W.D. Phillips, private communication.

[12] C. Orzel, A.K. Tuchman, M.L. Fenselan, M. Yasuda, M.A. Kasevich, Science 291, 2386 (2001).

[13] P. Morse, H. Feshbach, Methods of Theoretical Physics, McGraw-Hill, New York 1953. 
[14] B.P. Anderson, M.A. Kasevich, Science 282, 1686 (1998).

[15] Y.B. Band, B. Malomed, M. Trippenbach, submitted to Phys. Rev. A.

[16] Y.S. Kivshar, B.A. Malomed, Rev. Mod. Phys. 61, 763 (1989).

[17] C. Menotti, J.R. Anglin, J.I. Cirac, P. Zoller, Phys. Rev. A 63, 023601 (2001).

[18] Y.B. Band, M. Trippenbach, submitted to Phys. Rev. Lett. 